\title{
Crimean-Congo hemorrhagic fever in a dengue-endemic region: lessons for the future
}

\author{
Farheen Ali $^{1}$, Taimur Saleem ${ }^{2}$, Umair Khalid ${ }^{2}$, Syed Faisal Mehmood ${ }^{1}$, Bushra Jamil ${ }^{3}$ \\ ${ }^{1}$ Department of Medicine, Aga Khan University, Stadium Road, Karachi 74800, Pakistan \\ ${ }^{2}$ Medical College, Aga Khan University, Stadium Road, Karachi 74800, Pakistan \\ ${ }^{3}$ Departments of Medicine and Pathology and Microbiology, Aga Khan University, Stadium Road, Karachi 74800, \\ Pakistan
}

\begin{abstract}
Crimean-Congo hemorrhagic fever and dengue hemorrhagic fever are endemic in Pakistan. However, the overlap of geographic distribution and early clinical features between the two conditions make a reliable diagnosis difficult in the initial stage of illness. A 16-year-old boy presented with a history of hematemesis and high-grade fever. A preliminary diagnosis of dengue hemorrhagic fever was made and supportive treatment was instituted; however, the patient continued to deteriorate clinically. Dengue IgM antibody testing was negative on the third day of admission. Qualitative polymerase chain reaction test for Crimean-Congo hemorrhagic fever viral RNA was sent but the patient expired shortly after the results became available on the sixth day of admission. Considerable resources had to be expended on contact tracing and administration of ribavirin prophylaxis to all the health-care workers who had come in contact with the patient. It is crucial that Crimean-Congo hemorrhagic fever be recognized and treated at an early stage because of longer term financial and health implications for contacts such as health-care workers in the setting of a developing country. Increased surveillance of dengue and Crimean-Congo hemorrhagic fever cases is warranted for the derivation of reasonably reliable, cost-effective and prompt predictors of disease diagnosis. These predictors can help guide future decisions in the management of similar cases. Ultimately, such a strategy may translate into better cost containment in resource-poor settings. Institution of ribavirin prophylaxis in selected patients also merits consideration.
\end{abstract}

Key words: Crimean-Congo hemorrhagic fever, ribavirin, prophylaxis, thrombocytopenia

J Infect Dev Ctries 2010; 4(7):459-463.

(Received 4 January 2010 - Accepted 29 March 2010)

Copyright (c) 2010 Ali et al. This is an open-access article distributed under the Creative Commons Attribution License, which permits unrestricted use, distribution, and reproduction in any medium, provided the original work is properly cited.

\section{Introduction}

Crimean-Congo Hemorrhagic Fever (CCHF) virus (genus Nairovirus, family Bunyaviridae) is an enveloped virus with a genome consisting of three segments of single-stranded RNA of negative polarity [1]. This zoonotic virus is transmitted either through bite of the tick vector, mainly Hyalomma spp., or via direct contact with blood or tissues of viremic animals or humans [2]. The infection typically affects the reticuloendothelial system and causes activation of cytokine cascades in the body. This, in turn, leads to increased vascular permeability in the victims, ultimately culminating in hemorrhage and shock. As the disease evolves, multiple organ systems such as hematopoietic, neurologic, and pulmonary systems become involved $[3,4]$.

On the other hand, dengue virus belongs to the Flavivirus family and is transmitted through the bite of Aedes mosquitoes. The virus has four distinct serotypes. A person infected by one serotype develops life-long immunity to that particular serotype; however, infection by any of the other three seroptypes will have devastating consequences for that individual due to the phenomenon of "antibody enhancement" $[5,6]$. Dengue virus infection can manifest along a wide clinical spectrum including dengue fever (DF), dengue hemorrhagic fever (DHF) and dengue shock syndrome (DSS). DF patients present with fever, myalgias and morbilliform rash. In DHF, patients additionally have hemorrhagic manifestations while DSS patients have circulatory failure as a predominant feature [6]. The underlying pathophysiologic basis for the signs and symptoms of DHF and DSS is an increase in the permeability of the vasculature [5].

CCHF was first reported in the Crimea, Russia in 1944 [7]. Since then outbreaks have been described from various regions of the Middle East as well as Pakistan [8]. CCHF is endemic in Pakistan and multiple healthcare associated outbreaks have also 
been reported since the detection of the first case in 1976 [8]. Although outbreaks of CCHF in Pakistan have classically occurred in rural Balochistan, CCHF cases have also occurred in Karachi in recent times [1]. CCHF cases in Pakistan have mainly occurred in the months of October and November [1]. This time period roughly corresponds to the Islamic festival of Eid-ul-Azha, celebrated on days 10 to 13 of the last month of each lunar calendar year, when large flocks of sacrificial animals are brought into the cities from the rural areas of Pakistan [4].

DHF was first recognized in Asia in the 1950s [9]. The first major outbreak of DF in Karachi occurred in 1994 [5]. Since then this cosmopolitan city has experienced a few outbreaks of this arboviral fever, the most recent of which occurred in 20052006. During this epidemic, heavy morbidity and mortality burden was reported [10,11]. The case fatality rate incurred from $\mathrm{CCHF}$ has been reported to be considerably higher $(15-100 \%)$ as compared to DF (the case fatality rate for dengue shock syndrome and dengue hemorrhagic fever is around 3\%-5\% in Pakistan) [8,12-15].

The overlap of the geographic distribution and the similarity of the initial presentation of DF and CCHF make it difficult to reliably differentiate the two conditions, especially in the initial stages of the illness. Although conclusive, laboratory tests, particularly molecular-based tests, are not ubiquitously available in Pakistan. Such tests are also expensive and require time for completion. Patients, therefore, must be managed initially on clinical suspicion alone until laboratory results become available. However, this approach may sometimes have important subsequent implications when a definitive diagnosis becomes available. We describe here a case of CCHF in a young male who was initially managed on the suspicion of DHF. Considerable resources had to be expended later when a diagnosis of CCHF was confirmed in the same patient.

\section{Case history}

A 16-year-old male presented to the emergency department of Aga Khan University Hospital (AKUH) with a recent history of three episodes of large quantity hematemesis. He had also had intermittent, high-grade fever with chills and rigors for five days prior to presentation and multiple episodes of non-bilious vomiting and watery diarrhoea for two days. The remaining history obtained from the patient was unremarkable for tick bites, contact with animals, persons with similar illness, and recent or remote travel. He was a resident of Karachi, Pakistan.

At admission, he was febrile $\left(38.3^{\circ} \mathrm{C}\right)$ and moderately dehydrated. $\mathrm{He}$ was otherwise hemodynamically stable at that time. Physical examination did not reveal any cutaneous rash or active source of bleeding from mucocutaneous surfaces. Remaining general and systemic examinations were also unremarkable. Emergent management of the patient included fluid resuscitation and nasogastric (NG) tube placement. The aspirate from the tube revealed a large quantity of blood. Laboratory values at this time showed thrombocytopenia $\left(10 \times 10^{9} / \mathrm{L}\right)$, leucopenia $(2 \times$ $\left.10^{9} / \mathrm{L}\right)$, raised serum creatinine $(1.5 \mathrm{mg} / \mathrm{dl})$, and raised international normalized ratio (1.5) (Figure 1). A preliminary diagnosis of DHF was made and the patient was admitted into the special care unit (SCU). Fluid resuscitation continued and the initial management plan consisting of platelet transfusions and broad spectrum antibiotics was followed.

During the first three days of admission, the patient's vital signs deteriorated, particularly his blood pressure and pulse. In addition, his Glasgow coma scale (GCS) scores dropped dramatically. He was therefore intubated and transferred to the intensive care unit (ICU). His chest roentgenogram showed bilateral perihilar edema. He developed oliguria and his serum creatinine also showed serial increments. Dengue IgM antibody, which had been sent to the laboratory on the first day of admission, came back negative on the third day of admission. Elevated fibrin degradation products $(18 \mathrm{mg} / \mathrm{l})$ and D-dimer values (3.74 units) indicated development of consumption coagulopathy. Qualitative PCR for CCHF viral RNA was sent at this time in view of the patient's rapidly deteriorating clinical status and negative dengue serology.

Over the next few days, multi-organ failure developed rapidly and he was placed on continuous renal replacement therapy (CRRT). He also had an episode of atrial fibrillation with rapid ventricular rate (190-210 beats/min) requiring synchronized cardioversion on the sixth day of admission. PCR for CCHF viral RNA returned positive at this time. Before any specific measures could be instituted after the receipt of this result, the patient became bradycardiac and developed pulseless electrical activity (PEA). Cardiopulmonary resuscitation (CPR) code was initiated; however, it was stopped after 28 minutes of continuous resuscitative efforts. 
Figure 1. Serial measurements of the platelets and INR values of the patient during his hospital stay (a single value represents the average measurement of that parameter over 24 hours). Primary vertical axis shows INR values while the secondary vertical axis shows platelet count. Day 1 corresponds to the day of admission to the hospital.

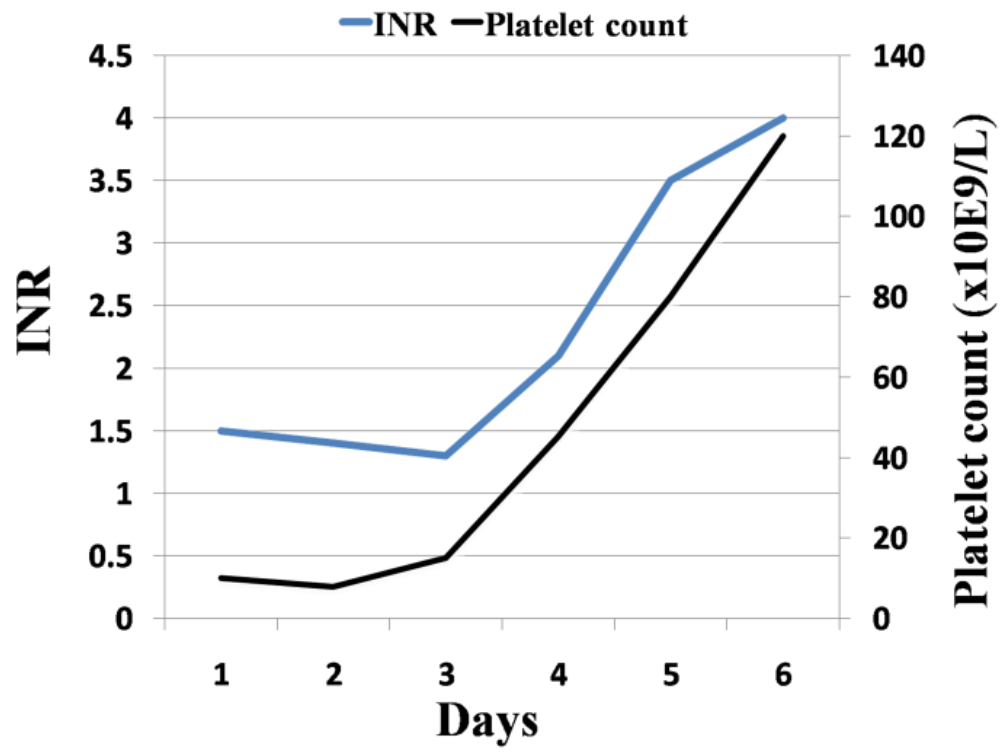

A meeting of the health-care team with the patient's family was immediately arranged. On directed questioning, they revealed that although two other members of the patient's family had experienced mild upper respiratory tract symptoms a few days ago, they had recovered uneventfully and did not have any current health complaints. They were counselled to visit the hospital in case any other family member developed symptoms.

Contact tracing of individuals in the hospital was also done. Seven doctors and 18 nurses were identified and given ribavirin prophylaxis for seven days. The total cost of this course for these individuals was US \$401. Some of these health-care professionals also reported adverse effects from the prophylactic treatment, including jaundice, nausea, and hair loss.

As soon as the patient had been identified as a case of CCHF, he was put in strict isolation per CCHF policy developed on the basis of the Centers for Disease Control and Prevention (CDC) recommendations. Prior to this, we were following only standard precautions. After the patient died, his body was bathed by the nursing staff, who ensured strict isolation in addition to contact and respiratory precautions. The cadaver was then carefully wrapped in a leak-proof bag. The family was also counselled about the hazards of bathing or exposing the body any further. They were advised to bury the body within the sealed bag.

\section{Discussion}

Infectious diseases still play a significant role in shaping the morbidity and mortality patterns in the developing countries [2]. Physicians in these areas stand at crossroads because of the endemic nature and non-specific overlapping features of many infectious diseases, such as tuberculosis, malaria, typhoid, and viral fevers. In Pakistan, viral hemorrhagic fevers (VHFs) such as CCHF are increasingly recognized as a public health problem with immense implications for the already limited health-care resources [2]. It is unfortunate that the understanding regarding clinical presentations and modes of spread of CCHF among health-care personnel in Pakistan is poor despite the endemic nature of the condition [16]. Similarly, knowledge about the basic principles of infection control among health-care personnel and hospital administrators is lacking [2]. Education of both the public as well as health-care professionals about viral hemorrhagic fevers should, therefore, be undertaken on an urgent footing.

The delay in reaching a diagnosis of CCHF may be due to the prudence of ruling out competing and commoner diagnoses as well as the reliance on effective laboratory testing in such cases. In addition, media has preferentially highlighted DF as an emerging health problem when compared to CCHF. As mentioned earlier, CCHF cases in Pakistan have been increasingly documented around the time of the Islamic festival of Eid-ul-Azha in recent years. This 
is possibly due to the large flocks of sacrificial animals that are brought into the cities from the rural areas of Pakistan. The animals are kept in open areas or private enclosures and are usually slaughtered within the first three days of this Islamic festival [4]. Although no formal study has been conducted to investigate the burden or presence of Hyalomma ticks in Karachi, such a study is certainly warranted in view of the increasing number of cases of $\mathrm{CCHF}$ being reported from the city. It is possible that CCHF in this particular case was not suspected initially because of lack of this temporal association. In addition, history of exposure to livestock or other animals and infected individuals may not always be readily available from patients [1]. This patient did not give any history of exposure to livestock or ticks and examination also did not reveal any tick bites. All these factors can compositely delay the diagnosis and do not bode well for a resource-poor country such as Pakistan as far as cost-containment is concerned.

There are considerable overlaps in the clinical presentations of both CCHF and DHF. Patients with $\mathrm{CCHF}$ and DHF are both young (mean age for both has been reported to be approximately 26 years). Both diseases occur more frequently in males $[1,14,15]$. CCHF cases are frequently encountered in the months of October and November in Pakistan. In comparison, DHF cases have been reported in the months of August, September and October $[1,15]$. This temporal distribution for CCHF has been explained on the basis of increased livestock movement into cities at the time of Eid-ul-Azha [4]. For DHF, the temporal association of these months to illness can be explained by the rains of the monsoon season in Pakistan in July and August every year, when pools of stagnant water serve as important breeding hubs for the Aedes mosquitoes [15].

Patients with CCHF generally present with fever, vomiting and bleeding from body orifices $(\geq 50 \%$ of cases); however, petechiae or echymoses are seen less commonly $(\sim 30 \%)[1,4]$. In comparison, patients with DHF present with chills/rigors, vomiting, diarrhoea, headaches and abdominal pain $[5,6,14]$. Up to $35 \%$ patients with DHF present with mucosal bleeding while a morbiliform rash is present in about $80 \%$ of patients [5]. Skin lesions seen in DF are usually generalized macular blanchable erythematous rash $(65 \%)$ followed by discrete petechial lesions (35\%) and palmar erythema (30\%) [17]. However, other studies from Pakistan have reported a lower incidence of skin rash (24\%) in patients with DHF.
The discrepancy has been explained on the basis of factors that make detection of the rash difficult, such as a short-lived course of the rash, lack of itching, and the dark skin of the local population [6]. Although this particular patient presented with hematemesis and no rash, a diagnosis of DHF was made initially on the basis of a negative history for tick bites and exposure to livestock.

Laboratory parameters in patients with DHF and $\mathrm{CCHF}$ are also generally non-specific as patients with both diseases demonstrate thrombocytopenia, leucopenia with lymphocytosis, deranged coagulation profiles, deranged liver function tests, and elevated serum creatinine $[1,4,5,13,15]$. Although patients with CCHF are more likely to have persistent thrombocytopenia [1] as compared to patients with DHF who experience a nadir in platelet count during hospital stay followed by improvement [6], this phenomenon needs to be investigated in future studies.

Although we did not administer ribavirin in this patient, one possible strategy to consider in the future is the institution of ribavirin prophylaxis in selected patients. The diagnosis of CCHF is usually dependant on laboratory evaluation that is not available until after a few days of admission. Ribavirin should be given to such selected patients in whom a diagnosis of CCHF is strongly suspected on the basis of clinical grounds alone or whose clinical features sufficiently overlap features with DHF and in whom dengue serology is inconclusive. This approach may help prevent mortalities in cases similar to ours in the future.

\section{Conclusion}

In summary, our case represents a complex clinical scenario whereby the patient was initially suspected of having DHF, a disease that does not require institution of specific measures such as contact isolation or use of oral anti-viral agents. However, the diagnosis of CCHF was established on the sixth day of admission. Although the patient died shortly thereafter, considerable resources were spent on not only tracking contacts who had been exposed by this time but also on administering post-exposure prophylaxis to such contacts. The diversion of hospital resources towards these measures represents a considerable degree of necessary disruption undertaken to prevent spread of the disease. Also, CCHF cases may generate considerable stress and fear among hospital staff and other contacts [12]. Previously, CCHF related deaths in health-care 
workers have been reported from Pakistan on a few occasions $[1,8,18]$.

It is vital for clinicians to recognize $\mathrm{CCHF}$ at an early stage. However, that is not always possible.. Improved education of health-care workers and improved surveillance of DF and CCHF should be ensured in our region so that we can devise reasonably reliable, cost-effective and prompt predictors of disease diagnosis on the basis of the collected data. As this case clearly demonstrates, one of the biggest challenges we face in the early recognition of $\mathrm{CCHF}$ is our location in a dengueendemic area.

\section{Acknowledgements}

We are thankful to Mr. Nausherwan Saleem, Year 3 Student of Accounting and Finance at Lahore University of Management Sciences (LUMS), Pakistan, for his help in the preparation of accompanying graphs for this report.

\section{References}

1. Jamil B, Hasan RS, Sarwari AR, Burton J, Hewson R, Clegg C (2005) Crimean-Congo hemorrhagic fever: experience at a tertiary care hospital in Karachi, Pakistan. Trans R Soc Trop Med Hyg 99: 577-84.

2. Smego RA Jr, Sarwari AR, Siddiqui AR (2004) CrimeanCongo hemorrhagic fever: prevention and control limitations in a resource-poor country. Clin Infect Dis 38: 1731-35.

3. Vorou R, Pierroutsakos IN, Maltezou HC (2007) CrimeanCongo hemorrhagic fever. Curr Opin Infect Dis 20: 495500.

4. Rai MA, Khanani MR, Warraich HJ, Hayat A, Ali SH (2008) Crimean-Congo hemorrhagic fever in Pakistan. J Med Virol 80: 1004-1006.

5. Butt N, Abbassi A, Munir SM, Ahmad SM, Sheikh QH (2008) Haematological and biochemical indicators for the early diagnosis of dengue viral infection. J Coll Physicians Surg Pak 18: 282-85.

6. Ahmed S, Ali N, Ashraf S, Ilyas M, Tariq WU, Chotani RA (2008) Dengue fever outbreak: a clinical management experience. J Coll Physicians Surg Pak 18: 8-12.

7. Casals J, Henderson BE, Hoogstraal H, Johnson KM, Shelokov A (1970) A review of Soviet viral hemorrhagic fevers, 1969. J Infect Dis 122: 437-53.

8. Athar MN, Khalid MA, Ahmad AM, Bashir N, Baqai HZ, Ahmad M, Balouch AH, Bashir K (2005) Crimean-Congo hemorrhagic fever outbreak in Rawalpindi, Pakistan,
February 2002: contact tracing and risk assessment. Am J Trop Med Hyg 72: 471-73.

9. Jamil B, Hasan R, Zafar A, Bewley K, Chamberlain J, Mioulet V, Rowlands M, Hewson R (2007) Dengue virus serotype 3, Karachi, Pakistan. Emerg Infect Dis 13: 182.

10. Riaz MM, Mumtaz K, Khan MS, Patel J, Tariq M, Hilal H, Siddiqui SA, Shezad F (2009) Outbreak of dengue fever in Karachi 2006: a clinical perspective. J Pak Med Assoc 59: 339-44.

11. Wasay M, Channa R, Jumani M, Zafar A (2008) Changing patterns and outcome of Dengue infection; report from a tertiary care hospital in Pakistan. J Pak Med Assoc. 58: 48889.

12. (2008) Dengue fever: a regular epidemic? J Pak Med Assoc 58: $1-2$.

13. Ali N, Usman M, Syed N, Khurshid M (2007) Haemorrhagic manifestations and utility of haematological parameters in dengue fever: a tertiary care centre experience at Karachi. Scand J Infect Dis 39: 1025-28.

14. Khan E, Siddiqui J, Shakoor S, Mehraj V, Jamil B, Hasan R (2007) Dengue outbreak in Karachi, Pakistan, 2006: experience at a tertiary care center. Trans R Soc Trop Med Hyg 101: 1114-19.

15. Riaz MM, Mumtaz K, Khan MS, Patel J, Tariq M, Hilal H, Siddiqui SA, Shezad F (2009) Outbreak of dengue fever in Karachi 2006: a clinical perspective. J Pak Med Assoc 59: 339-44.

16. Sheikh NS, Sheikh AS, Sheikh AA (2004) Knowledge, attitude and practices regarding Crimean-Congo haemorrhagic fever among healthcare workers in Balochistan. J Ayub Med Coll Abbottabad 16: 39-42.

17. Saleem K and Shaikh I (2008) Skin lesions in hospitalized cases of dengue Fever. J Coll Physicians Surg Pak 18: 60811.

18. Altaf A, Luby S, Ahmed AJ, Zaidi N, Khan AJ, Mirza S, McCormick J, Fisher-Hoch S (1998) Outbreak of CrimeanCongo haemorrhagic fever in Quetta, Pakistan: Contact tracing and risk assessment. Trop Med Int Health 3: 878882.

\section{Corresponding author}

Dr. Taimur Saleem

Medical College

Aga Khan University

Stadium Road

Karachi, Pakistan

Phone: +92-333-3198838

Email: taimur@gmail.com

Conflict of interests: No conflict of interests is declared. 